\title{
In-vitro AND In-silico CORRELATION STUDIES OF NATURAL AChE INHIBITORS: AN APPROACH TOWARDS ALZHEIMER'S DISEASE
}

\author{
Vasudev Pai ${ }^{1}$, K. S. Chandrashekar ${ }^{1}$, Aravind Pai $^{2}$, Anuraag Muralidharan ${ }^{3}$ \\ and M. Manjunath Setty ${ }^{1, \bigotimes}$ \\ ${ }^{1}$ Department of Pharmacognosy, Manipal College of Pharmaceutical Sciences, Manipal \\ Academy of Higher Education, Manipal, Karnataka, India-576104 \\ ${ }^{2}$ Department of Pharmaceutical Chemistry, Manipal College of Pharmaceutical Sciences, \\ Manipal Academy of Higher Education, Manipal, Karnataka, India-576104 \\ ${ }^{3}$ Department of Pharmaceutical Biotechnology, Manipal College of Pharmaceutical Sciences, \\ Manipal Academy of Higher Education, Manipal, Karnataka, India-576104 \\ Corresponding Email: \\ Corresponding Author: mm.setty@manipal.edu
}

\begin{abstract}
Alzheimer's disease(AD) is one of the damaging neurodegenerative disorders portrayed by the deterioration of cognizance. AChE is involved in the termination of impulse transmission by hydrolysis of acetylcholine. The inactivation of AChE by various inhibitory drugs increases the accumulation of neurotransmitters in the cortex of the brain and normalizes the disrupted neurotransmission. Ayurveda is one of the oldest systems of medicine that uses a variety of crude drugs for memory-related disorders. Ashwagandha and Brahmi are widely used in Ayurveda for various ailments and also as a memory enhancer. The aim of the present study is to understand the effect of hydroalcoholic extracts of Withania somnifera and Centella asiatica, and their markers like Withanolide-A and Asiaticoside on AChE inhibition by in-vitro and in-silico screening model. Both the enriched extracts and marker compounds have favorable inhibitory activity and proved the potential candidates in the management of memoryrelated disorders.
\end{abstract}

Keywords: Alzheimer's Disease, AChE Inhibition, Molecular Docking, Withania somnifera, Centella asiatica.

RASĀYAN J. Chem., Special Issue, 2021

\section{INTRODUCTION}

Alzheimer's disease is one of the most well-known types of dementia common in an elderly individual with the age group of 60 years and above. ${ }^{1}$ National Institute on Aging characterizes AD as a dynamic, irreversible decrease in memory and performing routine activities, disturbing time and space orientation, change in language and communication skills, abstract thinking and the incapable of carrying out simple calculations. Other symptoms include changes in personality and mood swings, upset judgment, behavior and psychological symptoms. The cognitive derangement in AD is not a part of "normal" aging but due to disease progression. The most significant feature of $\mathrm{AD}$ is dementia, a typical term used for a number of syndromes characterized by a decline in intellectual ability that interferes with daily activities and social connections. ${ }^{2}$ The American Psychiatric Association characterizes dementia as a dynamic deterioration in intellectual ability that interferes with social and occupational performance. There are mainly two types of dementia: a) reversible dementia, which is caused by some medicines, vitamin deficiency or infection by pathogens; b) irreversible, which is due to a progressive neurodegeneration. ${ }^{3}$

\section{Fact and Figures of the Alzheimer's Disease}

Alzheimer's disease was once treated as a rare disease, but it is now considered a significant healthcare threat to the public that affecting seriously elderly individuals worldwide and must be addressed. AD is characterized by massive neuronal damage, which causes signal disturbances between the neuron cells in 
the brain. ${ }^{4}$ Percentage of people with AD is increased by 2 folds with every 5 years of age, so about $1 \%$ of 60 years of age and about $30 \%$ of 85 -year-old people have the disease. WHO has estimated that around 44 million individuals are presently suffering from dementia throughout the globe, and the number will be increased to 66 million by 2030 and around 116 million by the end of $2050 .{ }^{5}$ It has been noticed that in $\mathrm{AD}$, around seven stages are described. In the first stage, a person has normal behavior. In the second stage, there is a minor memory collapse. In the third stage, there are confusions in names, and it is a borderline condition and is not necessarily lead to Alzheimer's disease. In the fourth stage, the patient faces, inability to think rationally and is "mild" AD. In the fifth stage, "moderate" Alzheimer's, the patient can't recall the names of family members and have a lot of confusion following restlessness. In the sixth stage, "moderately severe" Alzheimer's, the patient faces difficulty in doing routine activities and needs of personal care. In the seventh stage, there is speech disturbance, loss of coordination. The last stage of AD is death due to multiple accidents and hospitalization. ${ }^{6}$

\section{Acetylcholinesterase Inhibition}

Acetylcholinesterase inhibitors (AChEIs) are the best existing treatment as of now utilized to treat mild to moderate AD. AChEIs moderate the progression of AD by increasing the level of acetylcholine in the brain. ${ }^{7}$ It prevents the enzyme acetylcholinesterase from breaking down acetylcholine and increase the communication between neuron. The schematic mechanism of cholinesterase inhibitor is represented in Fig.-1.

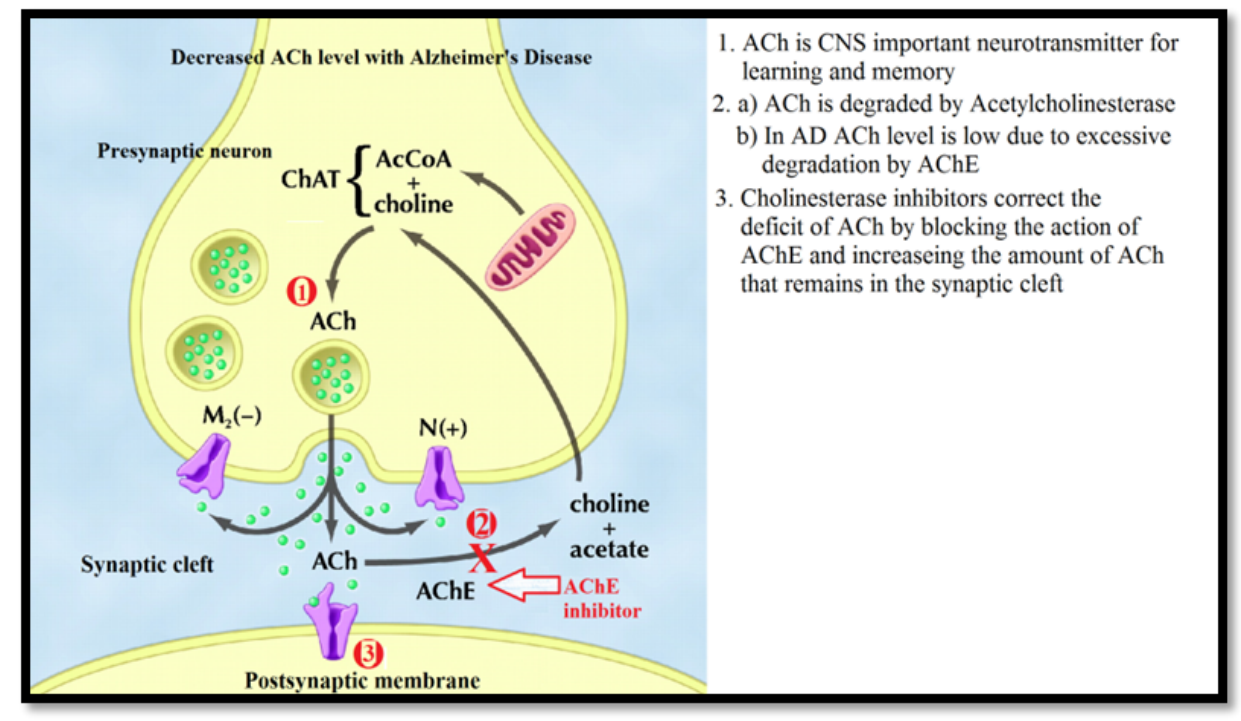

Fig.-1: Schematic Representation of the Mechanism of Cholinesterase Inhibitor

\section{Herbs reported to have Acetylcholinesterase Inhibition Activity Withania somnifera}

Ashwagandha is the common name for the plant Withania somnifera, which is also known as "Indian ginseng." It is well known in Ayurveda used to treat many disorders, including anxiety, depression, acute and chronic inflammation, fatigue, stress. It is also a very good nerve tonic and also beneficial in memory-related disorders. ${ }^{8}$ Ashwagandha plant belongs to the family Solanaceae, its root part is widely used as a medicinal herb. In Ayurveda, it is categorized as rasayana, which means rejuvenation. It also has strong antioxidant properties and has scavenging action on generated free radicals in the body. It is also known to strengthen and stimulate the immune system. Ashwagandha is known to reduce stress and thus calms the brain, particularly indicated by people with $\mathrm{AD}$ and other forms of dementia. ${ }^{9}$ Ashwagandha contains steroidal compounds, such as the steroidal lactones, which include withanolides A, with asomniferin A, and withanone etc. It also contains different amino acids, including tryptophan, a large amount of Iron. Some study says that neuronal cell death is triggered by amyloid plaques, withanolides blocks and clears the amyloid plaques. Aqueous extracts of roots of Ashwagandha have been found to increase acetylcholine in the brain of rats. ${ }^{10}$ 
RASĀYAN J. Chem.

83-91| Special Issue | 2021

\section{Centella asiatica}

It has a long history that entire herb is widely used as a memory-enhancing drug in Ayurveda. It is a wellknown revitalizer and strengthens nervous function and memory. C. asiatica is one of the Medhya (Nootropic) drugs in Ayurveda. ${ }^{11}$ Alcoholic extract of $C$. asiatica entire plant showed to have tranquilizing and cholinomimetic action due to its Pentacyclic triterpenoids saponins like asiaticoside, centelloside, madecassoside, brahmoside, brahminoside etc. The aqueous extract of the whole plant reported to increase the functioning of memory in rats was also studied. ${ }^{12}$ The extracts of Centella asiatica have antidepressant action by reducing the immobility time and also ameliorated imbalance in amino acid levels. A study demonstrated that $C$. asiatica also possesses potent antioxidant properties. ${ }^{13}$

\section{EXPERIMENTAL}

Plant material used: Withania somnifera and Centella asiatica were purchased from the local market. Candidate plants were identified and authenticated by taxonomist. Herbariums specimens of voucher no. PP535A and PP525B have been prepared respectively and deposited in the herbarium library of the Department of Pharmacognosy, Manipal College of Pharmaceutical Sciences, Manipal.

Extract preparation: The selected candidate plant materials were cleaned thoroughly, followed by drying in the shade for about 2 to 3 weeks. The dried plant material wares coarsely powdered and passed through sieve no. 22. The shade dried raw materials were extracted with hydroalcohol (80:20) by reflux condenser, concentrated using vacuum evaporator and lyophilized to powdery mass.

Markers used for the study: Withanolide-A and Asiaticoside and is procured from Natural Remedies Pvt. Ltd. Bengaluru.

\section{Acetylcholinesterase Inhibition Assay}

Amplex Acetylcholinesterase Assay Kit (A12217) was used for the study. It is an ultrasensitive screening method for in-vitro AChE inhibitory activity by a fluorescence microplate reader. In this assay, AChE activity is observed indirectly using 10-acetyl-3, 7-dihydroxyphenoxazine (Amplex Red reagent), a sensitive fluorogenic probe for Hydrogen peroxide. Primarily, AChE changes the acetylcholine substrate to choline. The converted choline is oxidized by choline oxidase to give betaine and hydrogen peroxide, which later, with the presence of horseradish peroxidase, reacts with Amplex Red reagent in a 1:1 stoichiometry and liberates highly fluorescent resorufin product. Resorufin has absorption and fluorescence emission maxima of approximately $571 \mathrm{~nm}$ and $585 \mathrm{~nm}$, respectively. ${ }^{14,15}$

\section{In-silico Molecular Docking Studies}

Molecular docking establishes an effective tool for screening and filtering compounds during drug discovery. In the long search for new drug discovery, it is feasible to find compounds with distinct features that can be potential lead molecules with effective therapeutic efficacy. Molecular docking studies can predict the appreciable orientations of a ligand or a molecule when interacting with targets such as enzymes/receptors to form a very stable complex to show a therapeutic effect. The key thermodynamic parameter involved in the process is binding free energy, which scans the theoretical stability between the ligand and complex protein of the enzyme or receptor. Based on the principles of molecular modeling, the primary goal is to identify the molecule that has AChE inhibition. ${ }^{16,17}$

All the molecular simulations studies were carried out on Schrodinger molecular modeling suite using Maestro interface. HP system with Pentium 4 Processor was used to run the simulations.

The molecular docking involves the following steps:

\section{Protein Preparation}

The Donepezil bound x-ray crystal structure of Acetylcholinesterase (protein databank with PDB ID: 4BTL) was extracted from PDB with a $2 \mathrm{~A}^{\circ}$ resolution. The protein preparation wizard refines the enzyme that involves two major steps, including preparation and refinement. The algorithm adds missing hydrogen, adds missing residue and caps terminals. It assigns bond orders. OPLS force field was used for energy minimization. The water molecules were removed in the region away from the active site. 
RASĀYAN J. Chem.

83-91| Special Issue | 2021

Disulphide bridges were created. Prime was used to fill missing chains and missing loops. Beyond 5å unit.

\section{Ligand Preparation}

Preparation of ligands was done by using Ligprep tool available with Schrodinger. OPLS 2005 force field was used. EPIK application was used to define the ionization states. Further, desalting was done to find the possible tautomers. Specified chirality's were fixed per ligand and were generated to find lowenergy ring conformations.

\section{Ligand Alignment}

Better docking poses alignment of the ligand, which is one of the prerequisites. Using flexible ligand alignment, the generated ligands were aligned.

\section{Glide Docking}

Docking is a two-stage process the receptor grid was defined in the first step. The actual docking of analogs to the receptors active site was performed in the next step. The ligand docking process uses Schrodinger's glide TM program (grid-based energetic ligand docking). Glide TM identifies the most significant interactions with a receptor, usually a protein, between one or more chemical entities. GlideTM has been operated in both rigid and flexible modes. The generated grid of the receptor was generated from the grid generation panel under the glide utility. The binding pockets electrostatic and Van der Waals potential was calculated using grid-based method. The actual docking of analogs on the binding site took place in the second step. The XP docking method option has been used for this purpose. The XP glide method semi-quantitatively ranks ligands ability to bind to the protein receptors bioactive conformation. ${ }^{18-20}$

\section{RESULTS AND DISCUSSION}

\section{Acetylcholinesterase Inhibition Assay}

The hydroalcoholic extracts of Ashwagandha and Brahmi were subjected for in-vitro AChE inhibition assay by using Amplex Assay Kit. Marker compounds Withanolide-A and Asiaticoside were also screened for AChE inhibition assay. The standard drug Rivastigmin is also screened and compared the effect with extracts and marker compounds. The results are given in Table-1.

Table 1. Acetylcholinesterase Inhibition Assay - IC50 Crude Extracts and Marker Compounds

\begin{tabular}{c|c}
\hline Extracts & IC50 Value \\
\hline Withania somnifera extract & $52.91 \mu \mathrm{g}$ \\
\hline Centella asiatica extract & $116.03 \mu \mathrm{g}$ \\
\hline Withanolide-A & $59.40 \mu \mathrm{g}$ \\
\hline Asiaticoside & $84.18 \mu \mathrm{g}$ \\
\hline Rivastigmin standard & $10.32 \mu \mathrm{M}$ \\
\hline
\end{tabular}

\section{In-silico Molecular Docking Studies of Various Markers for Acetylcholinesterase Inhibition}

Crystal ligand: Crystal ligand occupies the putative active site of the target protein AchE. Crystal ligand that fits in the hydrophobic binding cavity at the active site of AchE. The quaternary amino group Cation$\pi$ interaction with tyrosine 337 . There are 2 Cyclohexane rings was show $\pi$ - $\pi$ interaction with tyrosine 341 and tryptophan 286. The nitrate group showed hydrogen bond donor interaction with phenylalanine 295. The crystal ligand 2D interactions and crystal ligand 3D interactions Fig.-2 and Fig.-3.

\section{Withanolide-A}

The Withanolide-A showed a novel binding mode at the cleft region of the active site of acetyl cholinesterase. The planar steroidal ring system occupies the hydrophobic region at the putative target site. The carbonyl oxygen gives two prominent hydrogen bond acceptor interactions with the active site amino acids, namely, Tyrosine 72 and Aspartic acid 74, with a free energy of binding (docking score) of 10.408 The Withanolide-A 2D interactions and Withanolide-A 3D interactions Fig.-4. and Fig.-5. 
RASĀYAN J. Chem.

83-91| Special Issue | 2021

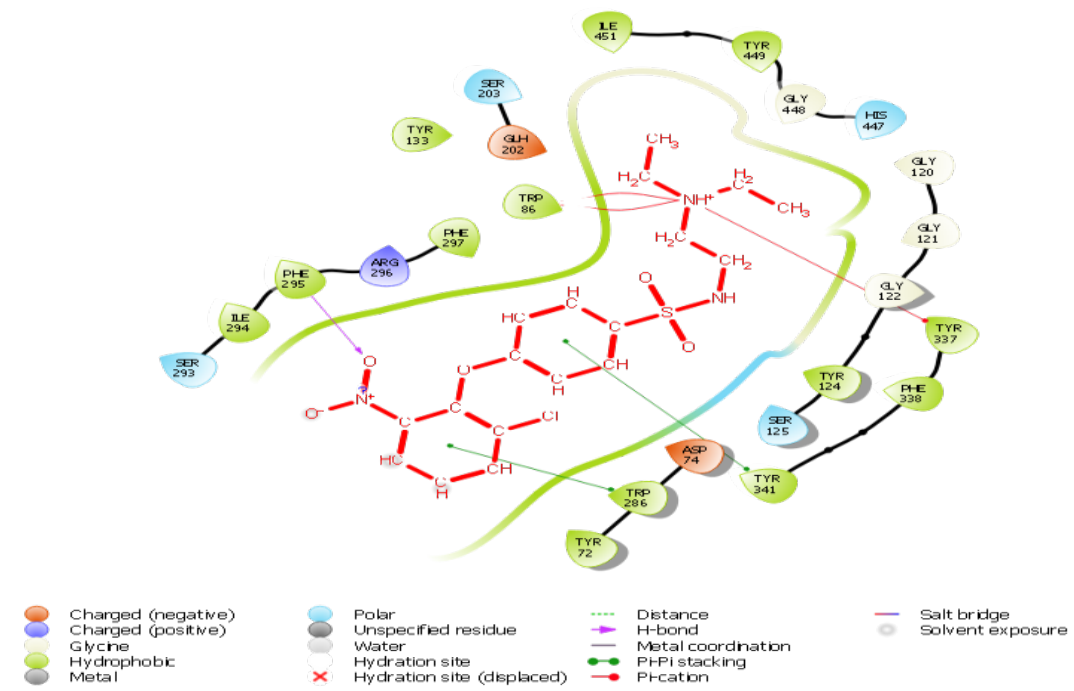

Fig.-2. Crystal Ligand 2D Interactions

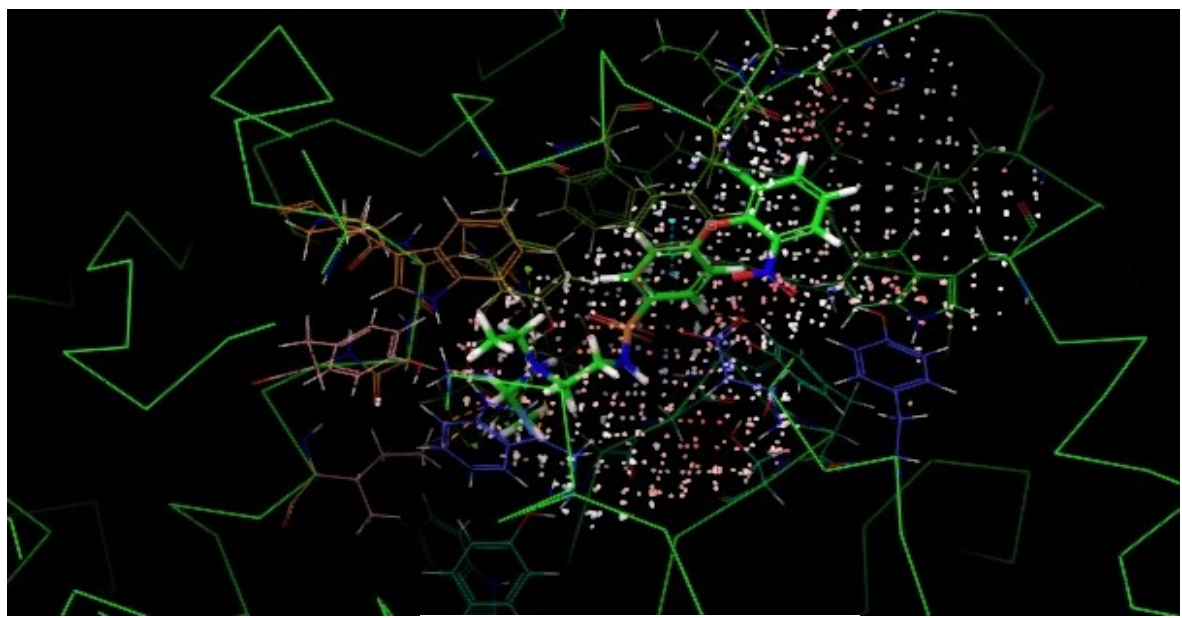

Fig.-3. Crystal Ligand 3D Interactions

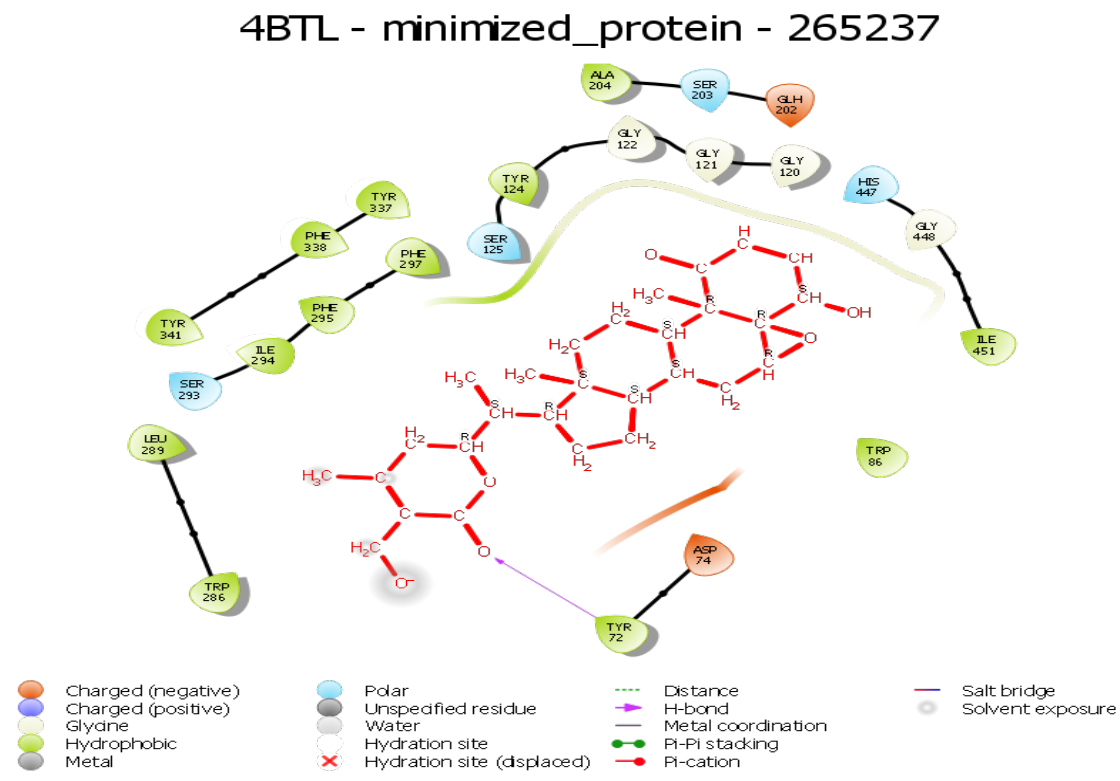

Fig.-4: Withanolide-A 2D Interactions 
RASĀYAN J. Chem.

83-91| Special Issue | 2021

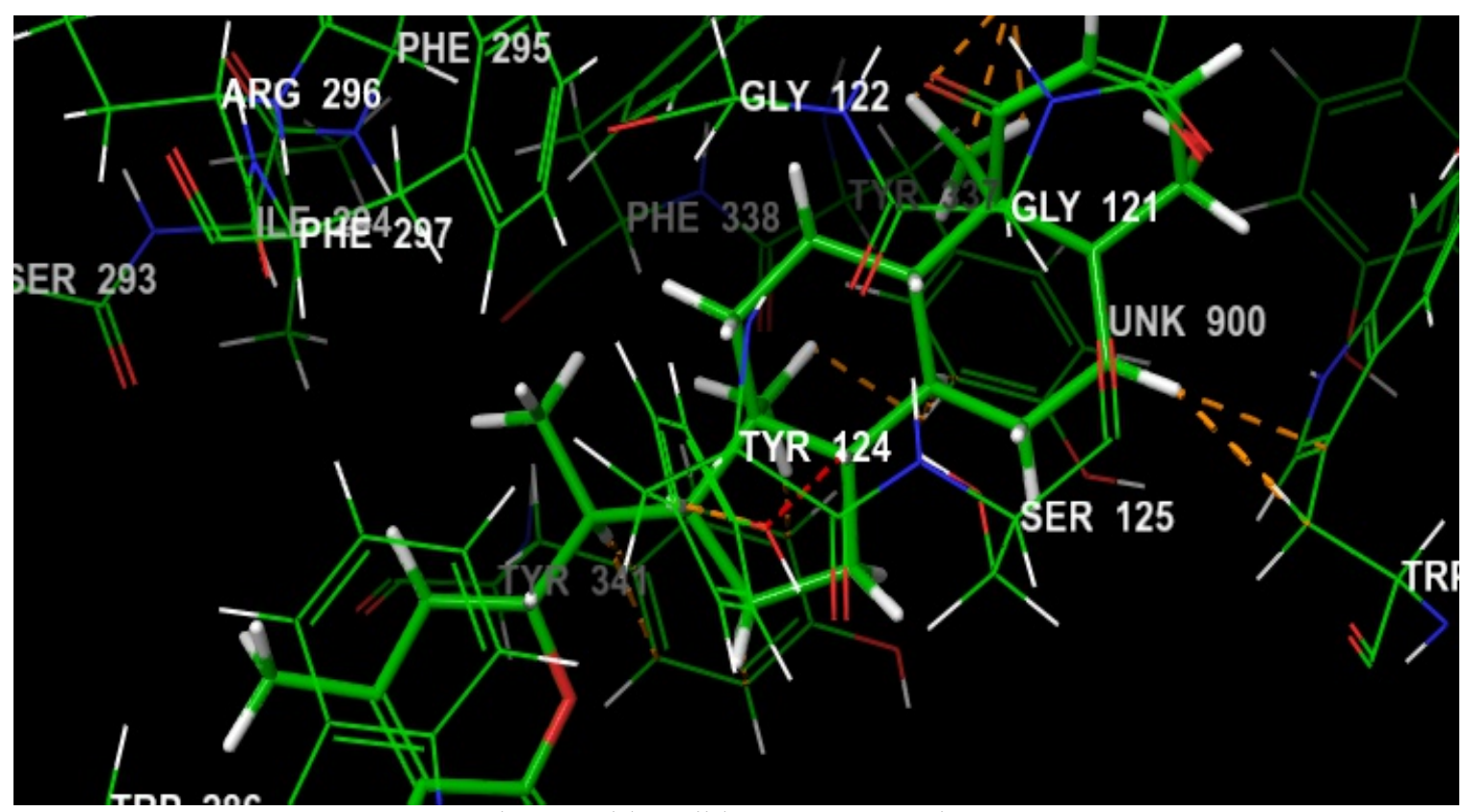

Asiatic Acid

Fig.-5: Withanolide-A 3D Interactions

The docking studies showed possible interactions of ligands at the active site of acetylcholinesterase. Asiatic acid occupies hydrophobic binding cavity at the active site of acetylcholinesterase. The polycyclic aliphatic system of Asiatic acid contains many rotatable bonds, which makes it flexible and facilitates the interactions with the active site of amino acids. Asiatic acid showed a docking score of -4.988 with the following interactions at the active site of acetylcholinesterase. Asiatic acid shows H-bond donor interaction with aspartic acid 283. The Asiatic acid 2D interactions and Asiatic acid 3D interactions Fig.-6 and Fig.-7.

4BTL - minimized - 119034

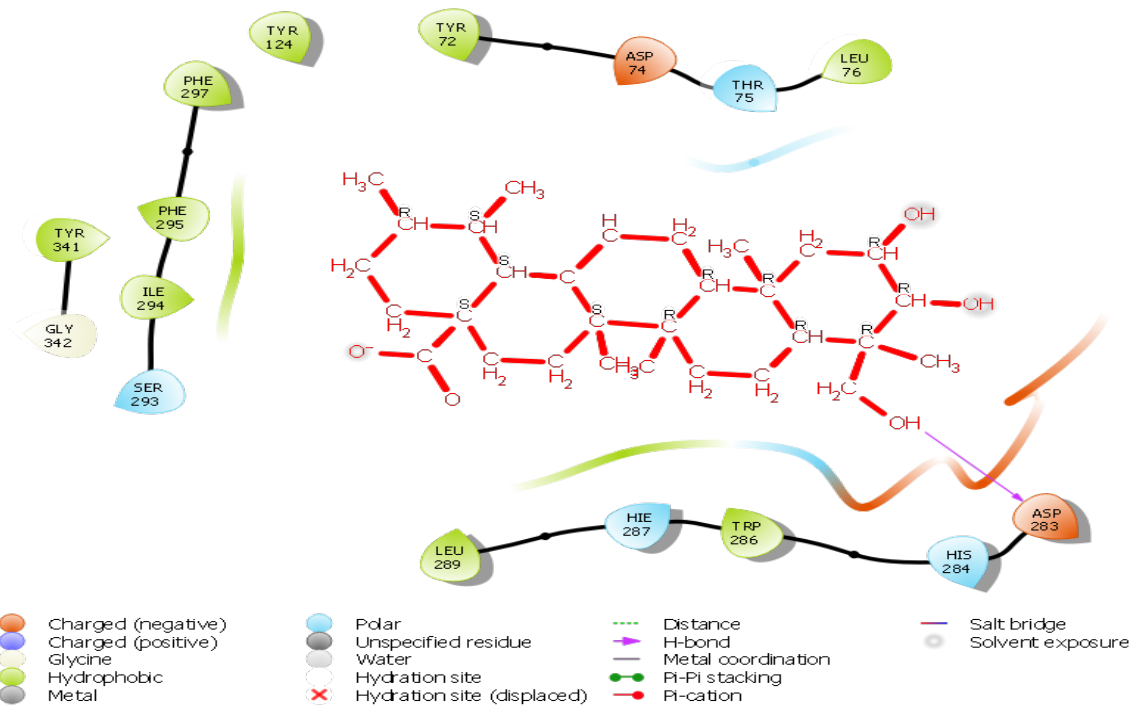

Fig.-6: Asiatic Acid 2D Interactions

\section{Donepezil}

The donepezil showed prominent ligand binding interactions at the putative site of acetylcholinesterase. It shows $\pi$-cation interaction with tryptophan 286 , carbonyl carbon has hydrogen bond acceptor interaction with phenylalanine 295 , cation- $\pi$ interaction of amino group with tyrosine 341 and phenyl ring $\pi-\pi$ interaction with tyrosine 124 with a docking score of -12.947 . The donepezil $2 D$ interactions and 
RASĀYAN J. Chem.

83-91| Special Issue | 2021

donepezil 3D interactions Fig.-8. and Fig.-9. The results of all in-silico studies are expressed in docking scores are given in Table-2.

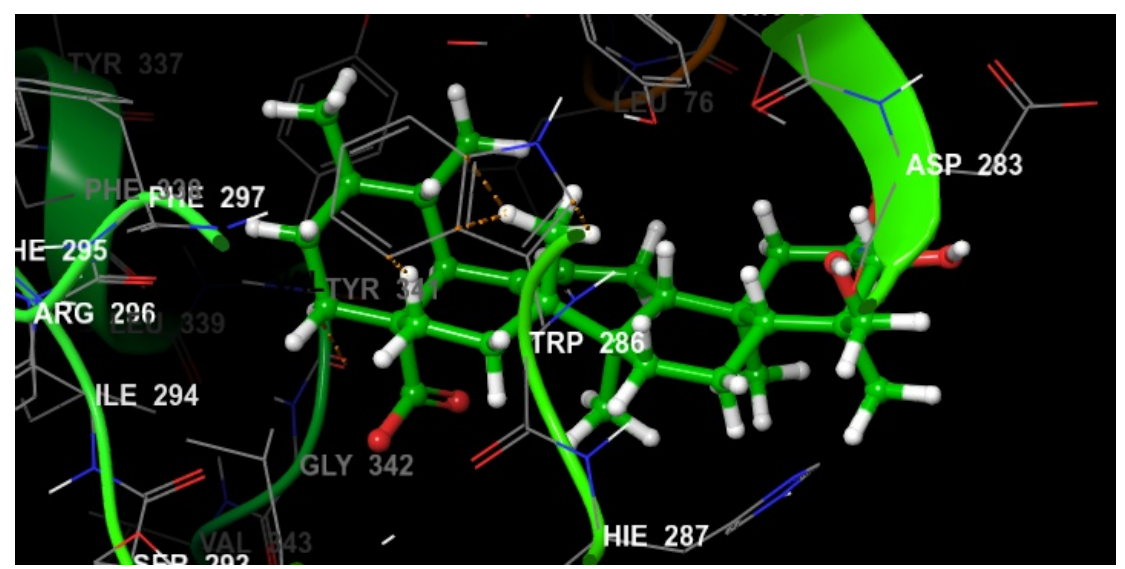

Fig.-7: Asiatic acid 3D Interactions

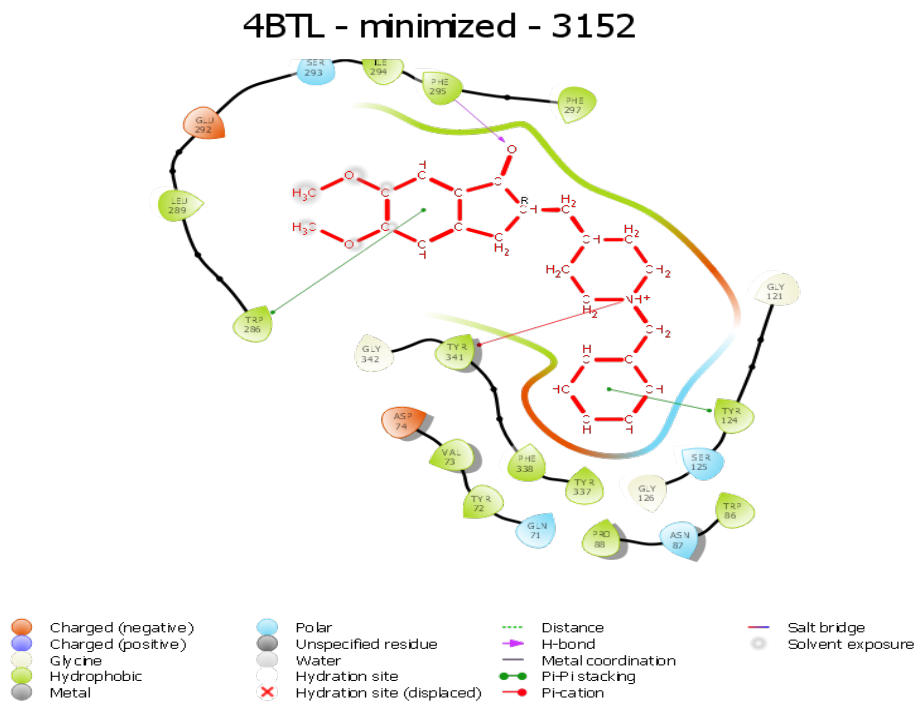

Fig.-8: Donepezil 2D Interactions

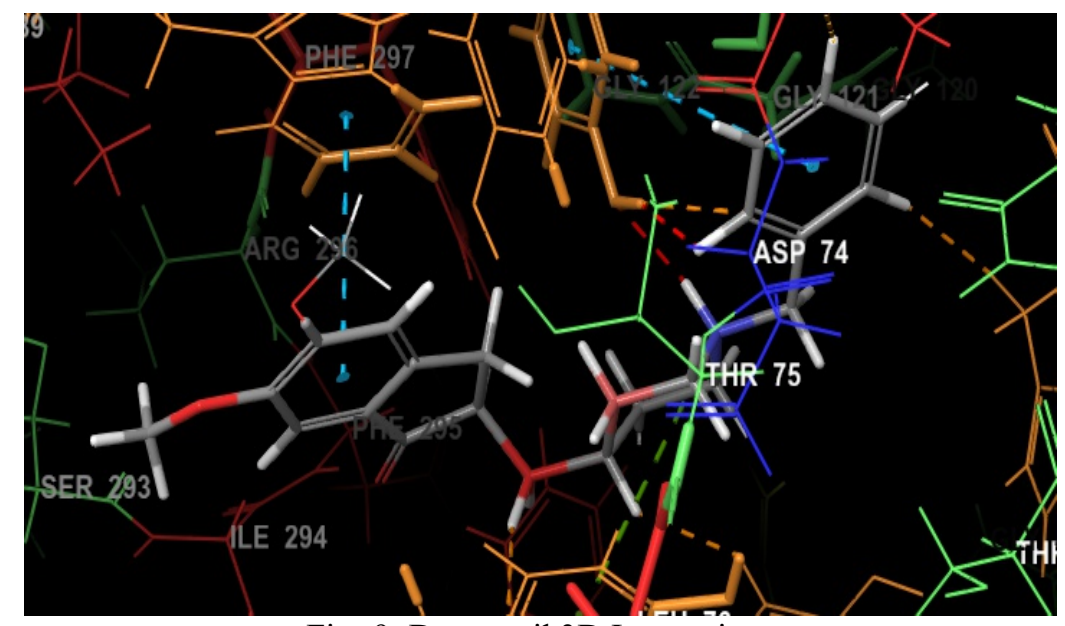

Fig.-9: Donepezil 3D Interactions 
RASĀYAN J. Chem.

83-91| Special Issue | 2021

Table-2: Docking Scores of Various Phytochemical Compounds

\begin{tabular}{c|c}
\hline Compound & Docking Scores \\
\hline Withanolide-A & -10.408 \\
\hline Asiatic acid & -4.988 \\
\hline Donepezil (Standard) & -12.947 \\
\hline
\end{tabular}

The cause of $\mathrm{AD}$ is due to atrophy of the brain cortex and is associated with disruption of cholinergic system in the brain cortex. An attempt has been made to screen extracts and markers for in-vitro AChE inhibition and IC50 value was determined. Withania somnifera extract shows $52.91 \mu \mathrm{g}$, Centella asiatica extract shows $116.03 \mu \mathrm{g}$, Withanolide-A shows $59.40 \mu \mathrm{g}$ and Asiaticoside shows $84.18 \mu \mathrm{g}$, whereas standard drug Rivastigmin shows $10.32 \mu \mathrm{M}$. As per IC50 values, Ashwagandha and Brahmi have significant $\mathrm{AChE}$ inhibition as compared to standard drugs. An attempt was also made to study in-silico molecular docking studies on the most important markers present in the extracts for AChE inhibition using Schrodinger molecular docking software and docking scores are compared with standard Donepezil. Withanolide-A and Asiatic acid show -10.408 and -4.988 , respectively, whereas standard Donepezil shows -12.947 .

\section{CONCLUSION}

The Alzheimer's pathology is very complex and believed to contain a deficit in cholinergic system that directly contribute to the disease progression. The current study was undertaken to understand the effect of traditional herbal drugs Ashwagandha and Brahmi. In-vitro and in-silico studies of extracts and their marker compounds suggest that both the drugs have significant AChE inhibition and can be used to control the symptoms of Alzheimer's disease.

\section{ACKNOWLEDGEMENT}

The authors are grateful to the Department of Pharmacognosy, Pharmaceutical chemistry and Pharmacology, Manipal College of Pharmaceutical Sciences, Manipal Academy of Higher Education for providing laboratory facilities and Schrödinger Centre for Molecular Simulation to carry out the work.

\section{REFERENCES}

1. D.A. Evans, H.H. Funkenstein, M.S. Albert, P.A. Scherr, N.R. Cook, M.J. Chown, L.E. Hebert, C.H. Hennekens and J.O. Taylor, Journal of the American Medical Association, 262(18), 2551(1989), https://doi:10.1001/jama.1989.03430180093036

2. J. Brown, G. Pengas, K. Dawson, L.A. Brown and P. Clatworthy, British Medical Journal, 338, b2023(2009), https://doi.org/10.1136/bmj.b2030

3. J.E. Graham, K. Rockwood, B.L. Beattie, R. Eastwood, S. Gauthier, H. Tuokko, I. McDowell, The Lancet, 349(9068), 1793(1997), https://doi.org/10.1016/S0140-6736(97)01007-6

4. N. Singh, B.R. Pandey and P. Verma, International Journal of Pharmaceutical Sciences and Drug Research 3(3), 162(2011), https://www.ijpsdr.com/index.php/ijpsdr/article/view/274.

5. A. Wimo and M. Prince, Alzheimer's Disease International World Alzheimer Report 2010 $\begin{array}{llll}\text { Alzheimer's } \quad \text { disease } & \text { International. }\end{array}$ https://www.alzint.org/u/WorldAlzheimerReport2010.pdf

6. The Progression of Alzheimer's disease, Alzheimer Society of Canada, 2008.

7. M. Mehta, A. Adem and M. Sabbagh, International Journal of Alzheimer's Disease, 1(2012), 8(2012) https://doi.org/10.1155/2012/728983

8. J.N. Dhuley, Phytotherapy Research, 15(6), 524(2001), https://doi.org/10.1002/ptr.874

9. R.V. Rao, O. Descamps, V. John and D.E. Bredesen, Alzheimer's Research \& Therapy, 4(3), 22 (2012), https://doi.org/10.1186/alzrt125

10. M.S. Parihar and T Hemnani, Journal of Biosciences, 28(1), 121(2003), https://doi.org/10.1007/BF02970142

11. V. Aparna, S.V. Mallya, P. Srikanth K.N and Sunil Kumar, The Journal of Phyto Pharmacology, 4(1), 1(2015)

12. K.J. Gohil, J.A. Patel and A.K. Gajjar, Indian Journal of Pharmaceutical Sciences, 72(5), 546(2010), https://doi.org/10.4103/0250-474X.78519

CORRELATION STUDIES OF NATURAL AChE INHIBITORS 
RASĀYAN J. Chem.

83-91| Special Issue | 2021

13. M.R. Sakina and P.C. Dandiya, Fitoterapia, 61(4), 291(1990)

14. O.R. Rana, P. Schauerte, R. Kluttig, J.W. Schröder, R.R. Koenen, C. Weber, K.W. Nolte, J. Weis, R. Hoffmann, N. Marx and E. Saygili, Autonomic Neuroscience, 156(1-2), 82(2010), https://doi.org/10.1016/j.autneu.2010.04.011

15. H.J. Kim, E.J. Shin, B.H. Lee, S.H. Choi, S.W. Jung, I.H. Cho, S.H. Hwang, J.Y. Kim, J.S. Han, C. Chung and C.G. Jang, Molecules and Cells, 38(9), 796(2015), https://doi.org/10.14348/molcells.2015.0116

16. P.A. Nogara, R.D. Saraiva, D. Caeran Bueno, L.J. Lissner, C. Lenz Dalla Corte, M.M. Braga, D.B Rosemberg, J.B. Rocha, BioMed Research International, (2015), 1(2015), http://dx.doi.org/10.1155/2015/870389

17. D.B. Kitchen, H. Decornez, J.R. Furr, J. Bajorath, Nature Reviews Drug Discovery, 3(11), 935(2004), https://doi.org/10.1038/nrd1549

18. P. Ambure, S. Kar and K. Roy, Biosystems, 1(116), 10(2014), https://doi.org/10.1016/j.biosystems.2013.12.002

19. G.D. Geromichalos, F.N. Lamari, M.A. Papandreou, D.T. Trafalis, M. Margarity, A. Papageorgiou, Z. Sinakos, Journal of Agricultural and Food Chemistry, 60(24), 6131(2012), https://doi.org/10.1021/jf300589c

20. Small-Molecule Drug Discovery Suite 2016-3: Glide, Version 7.2, Schrödinger, LLC, New York, NY, 2016.

[RJC-6602/2021] 